\title{
Efeitos do CPAP em indivíduos com AVC e condição clínica de apneia obstrutiva do sono
}

\author{
Effects of CPAP on individuals with stroke and clinical \\ condition of obstructive sleep apnea
}

\section{Efectos de la CPAP en personas con ACV y condición clínica de apnea obstructiva del sueño}

\author{
Josilaine Botelho Vasconcelos ${ }^{1}$, Júlia Bezan Castro Rodrigues dos \\ Santos $^{1}$, Vanessa Nascimento Venâncio ${ }^{1}$, Simone Cecilio Hallak \\ Regalo², Jacqueline Rodrigues de Freitas Vianna ${ }^{3}$, \\ Saulo Cesar Vallin Fabrin ${ }^{4}$
}

\begin{abstract}
1.Acadêmica de Fisioterapia no Claretiano Centro Universitário de Batatais, Batatais-SP, Brasil. 2.Professora Titular na Faculdade de Odontologia de Ribeirão Preto da Universidade de São Paulo (FORP/USP), Ribeirão Preto-SP, Brasil.

3.Professora Doutora no Claretiano Centro Universitário de Batatais, Batatais-SP, Brasil.

4.Professor Mestre no Claretiano Centro Universitário de Batatais, Batatais-SP, Brasil.
\end{abstract}

\begin{abstract}
Resumo
Introdução. O acidente vascular cerebral (AVC) é atualmente uma das principais causas de mortes e incapacidades no mundo, caracterizado por danos neurológicos que diminuem a qualidade de vida e, em muitos casos, provocam alterações respiratórias durante o sono, ocasionadas pela apneia obstrutiva do sono (AOS), sendo esta considerada um importante fator de risco. Diante disso, o objetivo do presente estudo é analisar a efetividade e os benefícios do uso da pressão positiva contínua nas vias aéreas (CPAP) em indivíduos com AVC que apresentam AOS. Método. Realizou-se uma revisão da literatura nas bases de dados Scielo, Bireme, PEDro, Medline e Pubmed, incluindo artigos publicados nos últimos dez anos, indexados na língua inglesa e portuguesa. Resultados. Os tratamentos com CPAP foram realizados com duração média de $4 \mathrm{~h} /$ noite, com PEEP de 2 a $12 \mathrm{cmH}_{2} \mathrm{O}$, sendo sua titulação realizada por oximetria de pulso, polissonografia ou por meio do AutoCPAP, apresentando redução nos índices de apneia-hipopneia, melhoria das escalas neurológicas e funcionais, com resultados significativos na qualidade do sono, eventos cardiovasculares e recorrências do AVC. Conclusão. O presente estudo sugere que o uso do CPAP em pacientes com AVC que possuem AOS é benéfico, sendo uma técnica eficaz nas comorbidades causadas por este distúrbio.

Unitermos. Acidente Vascular Cerebral; CPAP; Apneia do Sono; Reabilitação; Doenças Cardiovasculares
\end{abstract}

\footnotetext{
Abstract

Introduction. Stroke is currently a major cause of death and disability worldwide, characterized by neurological damage that reduces quality of life and, in many cases, causes respiratory changes during sleep, caused by obstructive sleep apnea (OSA), which is considered an important risk factor. Therefore, the aim of the present study is to analyze the effectiveness and benefits of using continuous positive airway pressure (CPAP) in individuals with stroke who have OSA. Method. A literature review was carried out in the Scielo, Bireme, PEDro, Medline, and Pubmed databases, including articles published in the last ten years, indexed in English and Portuguese. Results. CPAP treatments were performed with an average
} 
duration of $4 \mathrm{~h} /$ night, with PEEP from 2 to $12 \mathrm{cmH} 2 \mathrm{O}$, with titration performed by pulse oximetry, polysomnography or through AutoCPAP, with a reduction in apnea-hypopnea indexes, improvement in neurological scales and functional, with significant results in sleep quality, cardiovascular events and stroke recurrences. Conclusion. The present study suggests that the use of CPAP in stroke patients who have OSA is beneficial, being an effective technique in the comorbidities caused by this disorder.

Keywords. Stroke; Continuous Positive Airway Pressure; Obstructive Sleep Apnea; Rehabilitation; Cardiovascular Diseases

\section{Resumen}

Introducción. El accidente cerebrovascular (ACV) es actualmente una de las principales causas de muerte y discapacidad en el mundo, caracterizada por un daño neurológico que reduce la calidad de vida y, en muchos casos, provoca alteraciones respiratorias durante el sueño, provocadas por la apnea obstructiva del sueño (AOS), siendo esto se considera un factor de riesgo importante. Por lo tanto, el objetivo del presente estudio es analizar la efectividad y los beneficios del uso de presión positiva continua en las vías respiratorias (CPAP) en individuos con accidente cerebrovascular que padecen AOS. Método. Se realizó una revisión de la literatura en las bases de datos Scielo, Bireme, PEDro, Medline y Pubmed, incluyendo artículos publicados en los últimos diez años, indexados en inglés y portugués. Resultados. Los tratamientos de CPAP se realizaron con una duración media de 4h / noche, con PEEP de 2 a 12cmH2O, con titulación realizada por pulsioximetría, polisomnografía o mediante AutoCPAP, con reducción de los índices apnea-hipopnea, mejoría en escalas neurológicas y funcional, con resultados significativos en la calidad del sueño, eventos cardiovasculares y recurrencias de ictus. Conclusión. El presente estudio sugiere que el uso de CPAP en pacientes con ictus que tienen AOS es beneficioso, siendo una técnica eficaz en las comorbilidades provocadas por este trastorno.

Palabras clave. Accidente cerebrovascular; CPAP; Síndrome de la Apnea del Sueño; Rehabilitación; Enfermedades cardiovasculares

Trabalho realizado no Claretiano Centro Universitário de Batatais, Batatais-SP, Brasil.

\section{INTRODUÇÃO}

O acidente vascular cerebral (AVC) é atualmente uma das principais causas de incapacidades funcionais e óbitos no mundo ${ }^{1}$, com prevalência de 5 a 10 casos para cada 1000 habitantes. É caracterizado por danos neurológicos que podem ocorrer por uma obstrução ou hemorragia de um vaso sanguíneo cerebral, diminuindo a qualidade de vida e, em alguns casos, provocando alterações respiratórias durante 0 sono, ocasionadas pela apneia obstrutiva do sono (AOS) que esses indivíduos estão predispostos a desenvolver ${ }^{2,3}$. 
O seu tratamento tem como objetivo a prevenção de complicações e independência funcional para a realização das atividades de vida diária (AVD), prevenindo e reabilitando comorbidades provenientes ao AVC e abordando a AOS com cuidados que buscam evitar a recidiva de novos eventos e proporcionar a esses indivíduos melhoras neurocognitivas e funcionais ${ }^{4,5}$.

A AOS é recorrente em pessoas que sofreram AVC, sendo considerada um importante fator de risco, com prevalência nessa população de $38 \%$ a $70 \%$ e, quando não tratada, pode apresentar risco de novos AVCs ou até mesmo de morte ${ }^{6}$. Apesar de pouco conhecida, está associada a um maior tempo de internações hospitalares devido a fatores como a hipertensão arterial sistêmica, insuficiência cardiorrespiratória, hipertensão pulmonar, isquemia cardíaca, doença renal e diabetes. Além disso, a AOS ocasiona a piora da funcionalidade, resultando em maior dependência pós $\mathrm{AVC}^{7-10}$.

Independentemente do tipo de lesões neurológicas, a AOS possui uma importante relação com doenças cardiovasculares em conjunto com outros fatores, como sedentarismo, maus hábitos alimentares, alcoolismo, tabagismo, consumo de drogas hipnóticas, dislipidemia e tolerância reduzida à glicose ${ }^{11}$.

Indivíduos com AOS possuem respirações desordenadas durante o sono, onde ocorre a interrupção do fluxo de ar causado pelo colapso das vias aéreas superiores que acarretam aumento da pressão arterial, maior estresse 
oxidativo, comprometimento da função endotelial e ativação plaquetária. Além disso, a má qualidade de sono acaba prejudicando suas funções neurológicas, cognitivas e motoras ${ }^{5,12,13}$.

O tratamento mais utilizado na AOS em indivíduos com AVC é a pressão positiva contínua nas vias aéreas (CPAP), com a utilização de um aparelho que fornece um fluxo de ar por uma máscara facial, podendo esta ser nasal ou orofacial. $O$ equipamento permite a abertura da via aérea durante a inspiração e a expiração, proporcionando ao paciente uma respiração melhor durante o sono e, consequentemente, diminuição da sonolência diurna ${ }^{14}$, ajudando também na recuperação neurológica, cognitiva e dos sintomas depressivos $^{13}$. Além do mais, quando associada à reabilitação precoce, há melhora da função motora e das incapacidades, proporcionando uma melhor qualidade de vida e diminuição das disfunções causadas pelo $\operatorname{AVC}^{15,16}$.

Atualmente $\mathrm{O}$ uso de terapias alternativas para 0 tratamento da AOS podem não oferecer resultados eficazes como o uso do CPAP. Entretanto, apesar dos reconhecidos efeitos benéficos do seu tratamento, a adesão não é satisfatória, permanecendo estática nos últimos 15 anos em aproximadamente $34,1 \%$ dos acometidos ${ }^{17}$. Dentre os fatores que dificultam a adesão a longo prazo, estão os parâmetros do aparelho que é definido pela pressão expiratória final positiva (PEEP) em $\mathrm{cmH}_{2} \mathrm{O}$, temperatura, umidade, interface e máscara, dificultando o tratamento e interferindo diretamente nos seus resultados ${ }^{16}$. 
Outra falha considerável é que $70 \%$ a $80 \%$ dos indivíduos com AOS acabam não recebendo o diagnóstico. A deficiência física e a falta de acesso a polissonografia (PSG) dificultam ainda mais o diagnóstico para pacientes com AVC ${ }^{18}$.

Embora as diretrizes da American Heart Association/American Stroke Association recomendem que esses indivíduos realizem a triagem para AOS, não são todos que seguem tais recomendações e por consequência esses indivíduos não recebem um tratamento adequado ${ }^{19}$.

Diante da alta incidência, significativa recidiva de novos eventos, altos índices de mortalidade, danos neurocognitivos e comorbidades em indivíduos que sofreram AVC e possuem AOS, este estudo visa evidenciar o uso do CPAP e seus resultados no tratamento de tais pacientes.

\section{MÉTODO}

Para obter os resultados e respostas acerca da problematização apresentada neste estudo foram utilizadas as bases de dados PubMed, PEDro, Cochrane, Bireme e Scielo com artigos publicados entre os anos 2009 a 2019, indexados na língua inglesa e portuguesa. Os descritores utilizados para a pesquisa foram selecionados de acordo com a linguagem fornecida pelo Mesh, sendo eles: Stroke, Continuous Positive Airway Pressure, Obstructive Sleep Apnea e os seus correspondentes termos na língua portuguesa: Acidente Vascular Cerebral, Pressão Positiva 
Contínua nas Vias Aéreas e Apneia Obstrutiva do Sono. As palavras-chave foram combinadas utilizando os operadores booleanos AND e OR, sem restrição linguística

Para os critérios de inclusão, foram selecionados artigos originais referentes à pacientes que tiverem um evento anterior de acidente vascular cerebral e possuíam apneia obstrutiva do sono, utilizando o tratamento com pressão positiva contínua nas vias aéreas. Além disto, na presente revisão de literatura, os estudos identificados pela estratégia de busca deveriam consistir em ensaios clínicos randomizados, quase-randomizados ou prospectivos que utilizassem como intervenção fisioterapêutica o CPAP no tratamento da AOS.

Os critérios de exclusão foram artigos de revisão, estudos de caso e artigos que não possuíam o texto na íntegra. Foram excluídos também estudos que tratavam a AOS com métodos diferentes aos desejáveis para esta pesquisa. A partir dos artigos obtidos durante a pesquisa, foram avaliados os textos completos e as listas de referências bibliográficas isoladamente no intuito de identificar prováveis novos artigos para o processo de revisão.

\section{RESULTADOS}

Foram realizadas as buscas de artigos nas bases de dados eletrônicas por três avaliadores que conseguiram 
identificar 723 artigos publicados, após realizar análises dos resumos e títulos conforme demonstra a Figura 1.

Figura 1. Fluxograma de seleção dos ensaios clínicos pesquisados.
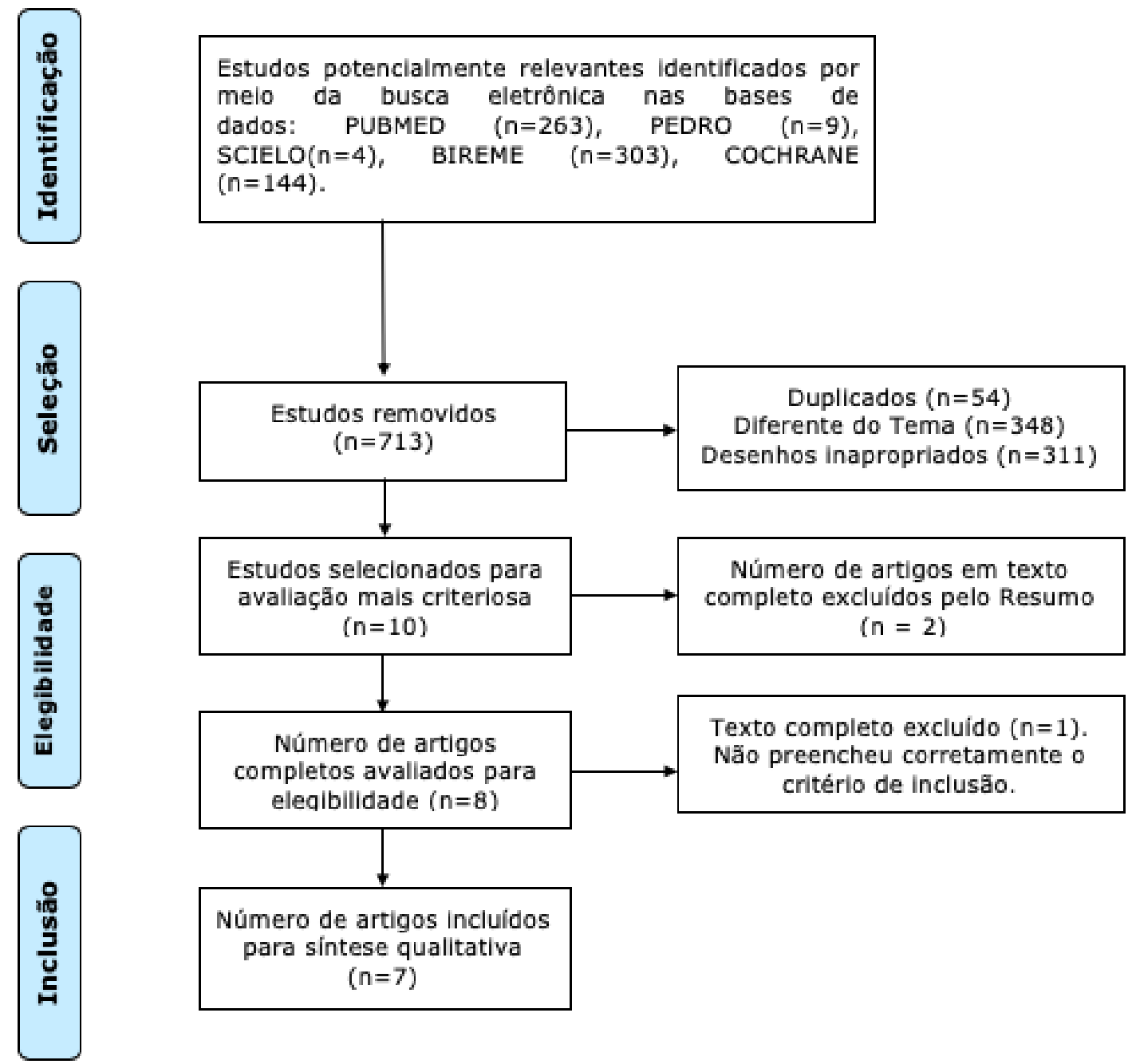

Texto completo excluído $(n=1)$. Não preencheu corretamente o critério de inclusão.

A revisão de literatura deste estudo identificou 7 publicações na língua inglesa relatando a aplicabilidade do CPAP em indivíduos com AVC que apresentam a AOS conforme demonstra as Tabelas 1 e 2 . 
Tabela 1. Dados referentes aos estudos que avaliaram o tratamento com CPAP e seus resultados.

\begin{tabular}{|c|c|c|c|c|c|}
\hline Autor & Objetivos & Estudo & Amostra & & Resultados \\
\hline Ren $2019^{16}$ & $\begin{array}{l}\text { Avaliar o papel do } \\
\text { CPAP como um fator } \\
\text { importante que afeta } \\
\text { o resultado após o } \\
\text { AVC }\end{array}$ & $\begin{array}{l}\text { Estudo } \\
\text { prospectivo } \\
\text { controlado } \\
\text { multicêntrico }\end{array}$ & $\begin{array}{l}\text { MF: } 128 \\
\text { Faixa } \\
\text { etária de36 } \\
\text { - } 80 \text { anos }\end{array}$ & $\begin{array}{l}0 \\
0 \\
0 \\
0\end{array}$ & $\begin{array}{l}\text { Melhora neurocognitiva; } \\
\text { Diminuição do índice de hipóxia } \\
\text { intermitente à noite; } \\
\text { Facilitação da melhora motora; } \\
\text { Redução de recorrência do } \\
\text { AVC; }\end{array}$ \\
\hline Kim $2019^{20}$ & $\begin{array}{l}\text { Determinar se o } \\
\text { tratamento com } \\
\text { CPAP melhora a } \\
\text { função ou cognição, } \\
\text { a qualidade do sono } \\
\text { e a sonolência diurna } \\
\text { em pacientes } \\
\text { hospitalizados que } \\
\text { apresentam AVC e } \\
\text { AOS }\end{array}$ & $\begin{array}{l}\text { Estudo } \\
\text { controlado } \\
\text { randomizado }\end{array}$ & $\begin{array}{l}\text { MF: } 40 \\
\text { Faixa } \\
\text { etária de } \\
18-80 \\
\text { anos }\end{array}$ & $\begin{array}{l}0 \\
\circ \\
\circ\end{array}$ & $\begin{array}{l}\text { Melhora na gravidade do AVC; } \\
\text { Melhora do equilíbrio e } \\
\text { marcha; } \\
\text { Diminuição da sonolência } \\
\text { diurna; } \\
\text { Progresso no domínio cognitivo } \\
\text { de atenção e cálculo, mas } \\
\text { nenhuma melhora significativa } \\
\text { nos outros domínios } \\
\text { cognitivos; }\end{array}$ \\
\hline $\begin{array}{l}\text { Gupta } \\
2018^{18}\end{array}$ & $\begin{array}{l}\text { Avaliar o efeito do } \\
\text { tratamento com } \\
\text { CPAP na prevenção } \\
\text { de novos eventos } \\
\text { vasculares em } \\
\text { pacientes com AVC } \\
\text { e AOS }\end{array}$ & $\begin{array}{l}\text { Estudo } \\
\text { controlado } \\
\text { randomizado }\end{array}$ & $\begin{array}{l}\text { M: } 70 \\
\text { Faixa } \\
\text { etária de } \\
43-71 \\
\text { anos }\end{array}$ & $\begin{array}{l}0 \\
0\end{array}$ & $\begin{array}{l}\text { Melhora funcional; } \\
\text { Ausência de resultados } \\
\text { significativos no desempenho } \\
\text { cognitivo; }\end{array}$ \\
\hline $\begin{array}{l}\text { Aaronson } \\
2016^{22}\end{array}$ & $\begin{array}{l}\text { Avaliar a eficácia } \\
\text { do tratamento com } \\
\text { CPAP em pacientes } \\
\text { com AVC durante } \\
\text { a reabilitação } \\
\text { hospitalar }\end{array}$ & $\begin{array}{l}\text { Estudo } \\
\text { controlado } \\
\text { randomizado }\end{array}$ & $\begin{array}{l}\text { MF:36 } \\
\text { Faixa } \\
\text { etária de42 } \\
-70 \text { anos }\end{array}$ & $\begin{array}{l}0 \\
0\end{array}$ & $\begin{array}{l}\text { Melhora da função; } \\
\text { Melhora significativa dos } \\
\text { domínios da atenção e } \\
\text { funcionamento executivo, } \\
\text { mas não nos demais } \\
\text { domínios cognitivos; }\end{array}$ \\
\hline Parra $2015^{7}$ & $\begin{array}{l}\text { Avaliar a influência } \\
\text { do tratamento com } \\
\text { CPAP nas recidivas } \\
\text { cardiovasculares e } \\
\text { na mortalidade em } \\
\text { pacientes com AVC } \\
\text { isquêmico e AOS } \\
\text { moderada a grave } \\
\end{array}$ & $\begin{array}{l}\text { Estudo } \\
\text { controlado } \\
\text { randomizado. }\end{array}$ & $\begin{array}{l}\text { MF: } 140 \\
\text { Faixa } \\
\text { estaria } \\
<75 \text { anos }\end{array}$ & 0 & $\begin{array}{l}\text { Melhora neurológica e } \\
\text { funcional; } \\
\text { Efeito positivo na sobrevida a } \\
\text { longo prazo; } \\
\text { Sobrevida cardiovascular } \\
\text { significativamente mais alta e } \\
\text { uma maior sobrevida livre de } \\
\text { eventos cardiovasculares; }\end{array}$ \\
\hline $\begin{array}{l}\text { Ryan } \\
2011^{15}\end{array}$ & $\begin{array}{l}\text { Analisar se o } \\
\text { tratamento da AOS } \\
\text { com o CPAP em } \\
\text { pacientes com AVC } \\
\text { melhora a } \\
\text { recuperação motora, } \\
\text { funcional e } \\
\text { neurocognitiva }\end{array}$ & $\begin{array}{l}\text { Estudo } \\
\text { randomizado } \\
\text { cego }\end{array}$ & $\begin{array}{l}\text { MF: } 44 \\
\text { Faixa } \\
\text { etária } \\
\text { de18-89 } \\
\text { anos }\end{array}$ & o & $\begin{array}{l}\text { Redução no índice de apneia- } \\
\text { hipopneia; } \\
\text { Aumento no escore da Escala } \\
\text { Neurológica Canadense; } \\
\text { Melhora motora e funcional, } \\
\text { mas sem resultados } \\
\text { significantes em relação a } \\
\text { cognição; }\end{array}$ \\
\hline $\begin{array}{l}\text { Parra } \\
2011^{21}\end{array}$ & $\begin{array}{l}\text { Determinar o } \\
\text { impacto do } \\
\text { tratamento com } \\
\text { CPAP em pacientes } \\
\text { com AVC isquêmico } \\
\text { acompanhados por } \\
2 \text { anos }\end{array}$ & $\begin{array}{l}\text { Estudo } \\
\text { prospectivo, } \\
\text { randomizado, } \\
\text { controlado e } \\
\text { multicêntrico }\end{array}$ & $\begin{array}{l}\text { MF: } 140 \\
\text { Faixa } \\
\text { etária }<75 \\
\text { anos }\end{array}$ & o & $\begin{array}{l}\text { Acelera a recuperação } \\
\text { neurológica; } \\
\text { Redução no índice de } \\
\text { mortalidade cardiovascular; } \\
\text { Sem diferenças } \\
\text { estatisticamente significativas } \\
\text { na sobrevida livre de eventos } \\
\text { cardiovasculares }\end{array}$ \\
\hline
\end{tabular}

CPAP - Pressão Positiva Continua nas Vias Aéreas; M - masculino, F - feminino. 
Tabela 2. Informações sobre como foi realizado o tratamento com CPAP.

\begin{tabular}{|c|c|c|c|c|c|c|}
\hline Autor & Duração & Tempo & Máscara & $\begin{array}{l}\text { Titulação e } \\
\text { Parâmetros }\end{array}$ & IAH & Escalas \\
\hline $\begin{array}{l}\text { Ren } \\
2019^{16}\end{array}$ & $\begin{array}{l}\text { Até } \\
24 \text { meses }\end{array}$ & $\geq 4 \mathrm{~h} /$ noite & Oronasal & $\begin{array}{l}\text { AutoCPAP; } \\
8 \pm 4 \mathrm{cmH}_{2} 0\end{array}$ & $\begin{array}{l}\text { Definido como número } \\
\text { médio de apneias e } \\
\text { hipopneias por hora }\end{array}$ & $\begin{array}{l}\text { NIHSS, FMA, } \\
\text { BI, MEEM, } \\
\text { HAMA, HRSD }\end{array}$ \\
\hline $\begin{array}{l}\mathrm{Kim} \\
2019^{20}\end{array}$ & 3 semanas & $>4 \mathrm{~h} /$ noite & $\begin{array}{l}\text { Nasal } \\
\text { e oronasal }\end{array}$ & $\begin{array}{l}\text { Titulação não } \\
\text { especificada; } \\
12 \mathrm{cmH}_{2} \mathrm{O}\end{array}$ & $\geq 20$ eventos/h & $\begin{array}{l}\text { NIHSSS, K- } \\
\text { MBI, EQ-5D e } \\
\text { MMSE }\end{array}$ \\
\hline
\end{tabular}

\begin{tabular}{|c|c|c|c|c|c|c|}
\hline $\begin{array}{l}\text { Gupta } \\
2018^{18}\end{array}$ & $\begin{array}{l}\text { Até } \\
12 \text { meses }\end{array}$ & $\begin{array}{l}4,2 \pm 1,32 \\
\text { h/noite }\end{array}$ & $\begin{array}{l}\text { Nasal } \\
\text { e oronasal }\end{array}$ & $\begin{array}{l}\text { PSG; } \\
\pm 2 \mathrm{~cm} \mathrm{H}_{2} \mathrm{O}\end{array}$ & $>15$ eventos/h & $\begin{array}{l}\text { BI, mRS, } \\
\text { Análise de } \\
\text { sobrevivência } \\
\text { de } \\
\text { Kaplan-Meier, } \\
\text { ESS }\end{array}$ \\
\hline $\begin{array}{l}\text { Aaronson } \\
2016^{22}\end{array}$ & $\begin{array}{l}4 \text { semanas } \\
\text { com } \\
\text { acompanha } \\
\text { mento por } 2 \\
\text { meses }\end{array}$ & 2,5h/noite & $\begin{array}{l}\text { Não } \\
\text { especificado }\end{array}$ & $\begin{array}{l}\text { Titulação por } \\
\text { oximetria noturna. } \\
\text { A pressão foi } \\
\text { ajustada até que o } \\
\text { ODI fosse reduzido } \\
\text { ao normal } \\
\text { (ODI<5). Se } \\
\text { houvesse falha em } \\
\text { reduzir } \\
\text { adequadamente o } \\
\text { ODI, o CPAP era } \\
\text { ajustado por PSG } \\
\text { para reduzir o IAH } \\
\text { para <5 ou para a } \\
\text { pressão mais alta } \\
\text { tolerada }\end{array}$ & $\geq 15$ & $\begin{array}{l}\text { CNS, NIHSS, } \\
\text { USER, SSS, } \\
\text { Checklist } \\
\text { Individual } \\
\text { Strength, } \\
\text { Hospital } \\
\text { Anxiety and } \\
\text { Depression } \\
\text { Scale e Sleep } \\
\text { Quality Scale }\end{array}$ \\
\hline $\begin{array}{l}\text { Parra } \\
2015^{7}\end{array}$ & 5 anos & $\begin{array}{l}\text { Média de } \\
5,3 \mathrm{~h} / \text { noite }\end{array}$ & Nasal & $\begin{array}{l}\text { AutoCPAP; } \\
8,6 \pm 1,5 \mathrm{~cm} \mathrm{H} \mathrm{H}_{2}\end{array}$ & $\geq 20$ eventos/h & $\begin{array}{l}\text { BI, Escala } \\
\text { Multifacetada, } \\
\text { CNS, mRS, } \\
\text { Questionário } \\
\text { SF-36 } \\
\end{array}$ \\
\hline $\begin{array}{l}\text { Ryan } \\
2011^{15}\end{array}$ & 4 semanas & $\begin{array}{l}\text { Média de } \\
4,96 \pm 2,25 \\
\text { h/noite }\end{array}$ & $\begin{array}{l}\text { Não } \\
\text { especificado }\end{array}$ & $\begin{array}{l}\text { PSG para reduzir o } \\
\text { IAH para }<5 \text { ou } \\
\text { para a pressão } \\
\text { mais alta tolerada } \\
8,1 \pm 0,5 \mathrm{cmH} 20 \\
\end{array}$ & $\geq 15$ eventos/h & CNS, TC6, \\
\hline $\begin{array}{l}\text { Parra } \\
2011^{21}\end{array}$ & $\begin{array}{l}23,04 \pm 3,7 \\
\text { meses }\end{array}$ & $\begin{array}{l}5,3 \pm 1,9 \\
\text { h/noite }\end{array}$ & Nasal & $\begin{array}{l}\text { AutoCPAP; } \\
8,6 \pm 1,5 \mathrm{cmH} 20\end{array}$ & $>20$ eventos/h & $\begin{array}{l}\text { BI, CNS, } \\
\text { Escala Rankin } \\
\text { e } \\
\text { SF-36 }\end{array}$ \\
\hline
\end{tabular}

AHI - Índice de apneia-hipopneia; ESS - Escala de Sonolência de Epworth; mRS - Escala de Rankin Modificada; FMA - Escala de Avaliação Fugl-Meyer; BI - Índice de Barthel; ; MEEM - Exame do Estado Minimal; HAMA - Escala de Ansiedade de Hamilton, HRSD - Escala de Depressão de Hamilton, NIHSS National Institute of Health Stroke; K-MBI - Versão coreana do Índice de Barthel Modificado; EQ-5D EuroQol-5 Dimension; ; K-MMSE - Mini-Mental State Examination ; ODI - Índice de Dessaturação de Oxigênio; CNS - Canadian NeurologicalScale, TC6 - Teste de Caminhada de 6 minutos; SSS - Escala de Stanford sleepiness e BDI - Inventario de Depressão de Beck-B; BBS - Escala de Equilíbrio de Berg. 


\section{DISCUSSÃO}

Atualmente a AOS é uma condição altamente prevalente em indivíduos com AVC, agravando o quadro clínico destes pacientes. De acordo com a prática baseada em evidência e nos estudos realizados sobre a AOS, é nítido que os sintomas decorrentes deste distúrbio são inúmeros. Além disso, está diretamente relacionada a outros problemas de saúde como cardiopatias, síndromes metabólicas e comprometimentos neurocognitivos, afetando de forma direta a qualidade de vida,16,21,22. O uso do CPAP busca minimizar estes sintomas e melhorar a qualidade de vida, atuando não somente na capacidade funcional e na sonolência diurna, mas em fatores como melhora na gravidade do AVC, redução do índice de apneia-hipopneia e mortalidade cardiovascular ${ }^{15,20,21 .}$

Porém, apesar do uso do CPAP ser considerado padrãoouro no tratamento da AOS, na literatura não há muitos estudos que comprovem seus efeitos em indivíduos que também tiveram um evento de acidente vascular cerebral. Mediante isto, buscando avaliar a eficácia deste recurso após o AVC e diminuir os sintomas decorrentes deste distúrbio, o tratamento com CPAP foi utilizado durante $4 \mathrm{~h} /$ noite $^{16}$, onde foi observada a redução da recorrência do AVC e melhora da função motora que se encontrava prejudicada devido a hemiplegia, impossibilitando que os pacientes realizassem suas atividades de vida diária.

Este resultado também foi encontrado em outro estudo $^{19}$ que utilizou o CPAP com média de $4 \mathrm{~h} /$ noite, onde 
foi igualmente observado um aumento da capacidade funcional. Além da melhora funcional, também foi observado que o grupo CPAP, quando comparado ao grupo controle, obteve melhora no índice de apneia-hipopneia e aumento na saturação mínima da oxihemoglobina no sono ${ }^{15}$.

Foi constatada melhora da marcha e do equilíbrio em pacientes que também fizeram uso do CPAP durante 4h/noite, juntamente com uma melhora na gravidade do $A C^{20}$. Essa melhora funcional pode ser justificada devido a diminuição da sonolência diurna, que poderia ser um efeito benéfico na melhora cognitiva destes indivíduos.

Em outro estudo, foi possível observar que o tratamento precoce com CPAP na AOS em indivíduos com AVC isquêmico agudo foi benéfico ${ }^{21}$. Assim como nos estudos apresentados anteriormente, foi observada uma melhora significativa na funcionalidade desses indivíduos, que foi avaliada por meio da escala de Rankin. Ao comparar o grupo controle com o grupo CPAP, houve também uma diminuição da mortalidade cardiovascular, melhora nas avaliações neurológicas um mês depois do AVC e resultados significativos na escala Canadense nos pacientes que utilizaram o tratamento com CPAP. Um estudo posterior, encontrou em seus resultados efeitos significantes na sobrevida a longo prazo em indivíduos que possuíam AVC isquêmico com o uso do CPAP, além de benefícios neurológicos ${ }^{7}$. Também foi observado a diminuição de eventos cardiovasculares ao iniciar o tratamento precocemente. 
Os resultados positivos em relação a melhora funcional destes indivíduos, também foi observado no grupo CPAP com uma melhora significativa após o período de intervenção de quatro semanas ${ }^{22}$. Além disso, ainda comparando o grupo controle com o grupo CPAP, foi obtida uma melhora nos domínios da atenção e funcionamento executivo.

No entanto, na presente revisão de literatura não foram citados e nem encontrados na literatura trabalhos que esclarecessem uma significativa melhora cognitiva em indivíduos que sofreram AVC e possuem AOS, indicando que novos estudos devem ser realizados. Além disso, uma limitação frequente para a análise dos efeitos do CPAP nesses pacientes é a baixa adesão.

De acordo com os resultados apresentados na presente revisão, a eficácia do CPAP no tratamento da AOS é melhor do que a adquirida com terapias alternativas. No entanto, apesar de ser considerada uma das formas de tratamento mais eficaz para estes indivíduos, sua adesão é muito variável. Este detalhe é, sobretudo, relevante, uma vez que, como qualquer método de tratamento, os resultados benéficos e sua eficácia estão diretamente relacionados com a adesão.

Mediante essas considerações, podemos observar a importância de investir nesta técnica e analisar os motivos que influenciam na sua adesão, concentrando-se em melhorá-la e educar os pacientes e seus familiares sobre a terapia com CPAP. Fatores como o tipo de máscara utilizada, a pressão em $\mathrm{cmH}_{2} \mathrm{O}$, a disponibilidade do aparelho e o 
incentivo da família estão entre os principais motivos da baixa adesão.

De acordo com os trabalhos utilizados nesta revisão, a melhora cognitiva em indivíduos com AOS após um evento de AVC que fizeram uso do CPAP ainda não foi esclarecida, mas outros ensaios clínicos randomizados e o presente estudo apoiam o uso desta terapia, demonstrando os efeitos positivos que podem ser adquiridos com esta técnica e tornando-a uma opção eficaz de tratamento adicional para a melhoria da cognição e função nestes indivíduos.

\section{CONCLUSÃO}

Com a análise dos resultados encontrados, observa-se que o uso do CPAP, com titulação realizada por oximetria de pulso, polissonografia ou por meio do AutoCPAP, com duração média de tratamento de $4 \mathrm{~h} /$ noite, com nível de PEEP variando nos estudos de 2 a $12 \mathrm{cmH} 2 \mathrm{O}$, proporciona redução dos índices de apneia-hipopneia, dos eventos cardiovasculares e das recorrências do AVC, mostrando-se eficaz na melhora da qualidade do sono e nas alterações neurofuncionais, do equilíbrio e da marcha. Portanto, o tratamento com essa terapia é indicado para as disfunções ocasionadas pela AOS nesses indivíduos.

\section{REFERÊNCIAS}

1.Feigin VL, Forouzanfar MH, Krishnamurthi R, Mensah GA, Connor $H$, Bennett $A D$, et al. Global and regional burden of stroke during 1990-2010: findings from the Global Burden of Disease Study 2010. 
Lancet

$6736(13) 61953-4$

2.Terranova TT, Albieri FO, Almeida MD, Ayres DVM, Cruz SF, Milazzotto MV, et al. Acidente vascular cerebral crônico: reabilitação. Acta Fisiatr 2012;19:50-9. http://doi.org/10.5935/01047795.20120011

3.Dong JY, Zhang YH, Qin LQ. Obstructive sleep apnea and cardiovascular risk: Meta-analysis of prospective cohort studies. Atherosclerosis 2013;229:489-95. https://doi.org/10.1016/j.atherosclerosis.2013.04.026

4. Winstein CJ, Stein J, Arena R, Bates B, Cherney LR, Cramer SC, et al. Guidelines for Adult Stroke Rehabilitation and Recovery. Stroke 2016;47:e98-169. https://doi.org/10.1161/STR.0000000000000098

5.Loke YK Brown JW, Kwok CS, Niruban A, Myint PK. Association of obstructive sleep apnea with risk of serious cardiovascular events: a systematic review and meta-analysis. Circul Cardiovasc Qual Outcomes 2012;5:720-8. https://doi.org/10.1161/CIRCOUTCOMES.111.964783 6.Javaheri S, Barbe F, Campos-Rodriguez F, Dempsey JA, Khayat $\mathrm{R}$, Javaheri $\mathrm{S}$, et al. Sleep Apnea: Types, Mechanisms, and Clinical Cardiovascular Consequences. J Am Col Cardiol 2017;69:841-58.

https://doi.org/10.1016/j.jacc.2016.11.069

7.Parra O, Sánchez-Armengol A, Capote F, Bonnin M, Arboix A, Campos-Rodríguez, et al. Efficacy of continuous positive airway pressure treatment on 5-year survival in patients with ischaemic stroke and obstructive sleep apnea: a randomized controlled trial. J Sleep Res 2015;24:47-53. https://doi.org/10.1111/jsr.12181

8. Menon D, Sukumaran S, Varma R, Radhakrishnan A. Impact of obstructive sleep apnea on neurological recovery after ischemic stroke: A prospective study. Acta Neurol Scand 2017;136:419-26.

https://doi.org/10.1111/ane. 12740

9.Pinto JA, Ribeiro DK, AFS, Duarte C, Freitas GS. Comorbidities Associated with Obstructive Sleep Apnea: a Retrospective Study. Int Arch Otorhinolaryngol 2016;20:145-50. https://doi.org/10.1055/s0036-1579546

10.Xu S, Wan Y, Xu M, Ming J, Xing Y, An F, et al. The association between obstructive sleep apnea and metabolic syndrome: a systematic review and meta-analysis. BMC Pulmon Med 2015;105:15. http://doi.org/10.1186/s12890-015-0102-3

11.Sharma S, Culebras A. Sleep Apnea and Stroke. Stroke Vasc Neurol 2016;1:185-91. http://doi.org/10.1136/svn-2016-000038

12.Labarca G, Cruz NR, Descalzi F. Multisystemic involvement in obstructive sleep apnea. Rev Med Chile;142:748-57.

http://doi.org/10.4067/S0034-98872014000600009

13.Dharmakulaseelan L, Kirolos N, Kamra M, Armesto-Heys A, Bouthillier C, Runions S, et al. Educating Stroke/TIA Patients about Obstructive Sleep Apnea after Stroke: A Randomized Feasibility Study. J Stroke Cerebrovasc Dis 2019;28:104317. https://doi.org/10.1016/j.jstrokecerebrovasdis.2019.104317 
14.Patel N, Raissi A, Elias S, Kamra M, Kendzerska T, Murray BJ, et al. A Modified Definition for Obstructive Sleep Apnea in Home Sleep Apnea Testing after Stroke or Transient Ischemic Attack. J Stroke Cerebrovasc Dis 2018;27:1524-32.

https://doi.org/10.1016/j.jstrokecerebrovasdis.2017.12.052

15.Ryan CM, Bayley M, Green R, Murray BJ, Bradley TD. Influence of continuous positive airway pressure on outcomes of rehabilitation in stroke patients with obstructive sleep apnea. Stroke 2011;42:1062-7. http://doi.org/10.1161/STROKEAHA.110.597468

16. Ren L, Wang K, Shen H, Xu Y, Wang J, Chen R. Effects of continuous positive airway pressure (CPAP) therapy on neurological and functional rehabilitation in Basal Ganglia Stroke patients with obstructive sleep apnea: A prospective multicenter study. Medicine (Baltimore) 2019;98:e16344. http://doi.org/10.1097/MD.0000000000016344

17. Kendzerska T, Wilton K, Bahar R, Ryan CM. Short- and long-term continuous positive airway pressure usage in the post-stroke population with obstructive sleep apnea. Sleep Breat 2019;23:123344. https://doi.org/10.1007/s11325-019-01811-9

18.Gupta A, Shukla G, Afsar M, Poornima S, Pandey RM, Goyal V, et al. Role of Positive Airway Pressure Therapy for Obstructive Sleep Apnea in Patients with Stroke: A Randomized Controlled Trial. J Clin Sleep Med 2018;14:511-21. https://doi.org/10.5664/jcsm.7034

19. Winstein CJ, Stein CJ, Arena VCR, Bates B, Cherney IR, Steven C, et al. Guidelines for Adult Stroke Rehabilitation and Recovery: A Guideline for Healthcare Professionals from the American Heart Association/American Stroke Association. Stroke 2016;47:98-169. https://doi.org/10.1161/STR.0000000000000098

20.Kim H, Im S, Park JI, Kim Y, Sohn MK, Jee S. Improvement of Cognitive Function after Continuous Positive Airway Pressure Treatment for Subacute Stroke Patients with Obstructive Sleep Apnea: A Randomized Controlled Trial. Brain Sci 2019;9:252. https://doi.org/10.3390/brainsci9100252

21.Parra O, Sánchez-Armengol A, Bonnin M, Arboix A, CamposRodríguez $\mathrm{F}$, Pérez-Ronchel $\mathrm{J}$, et al. Early treatment of obstructive apnea and stroke outcome: a randomized controlled trial. Eur Respirator J 2011;37:1128-36.

https://doi.org/10.1183/09031936.00034410

22.Aaronson JA, Hofman WF, Van Bennekon CA, Van Bezeij T, Van Den Aardweg JG, Groet E, et al. Effects of Continuous Positive Airway Pressure on Cognitive and Functional Outcome of Stroke Patients with Obstructive Sleep Apnea: A Randomized Controlled Trial. J Clin Sleep Med 2016;12:533-41. https://doi.org/10.5664/jcsm.5684 